\section{Protección solar en el paciente pediátrico}

\section{Sun protection in pediatric patients}

Se denomina fotoprotección al conjunto de medidas que se pueden instaurar con el fin de proteger a la piel de la agresión producida por la exposición a los rayos solares. Incluye medidas físicas de evitación y el uso de fotoprotectores con el objetivo de prevenir posibles daños de la radiación ultravioleta en la piel. ${ }^{1}$

\section{RADIACIÓN ULTRAVIOLETA}

El 5\% de la luz solar que alcanza la tierra corresponde a radiación ultravioleta (290 a 400 nm); de acuerdo con su longitud de onda se le clasifica en UVA, UVB y UVC. La capa de ozono bloquea por completo la radiación UVC (100-290 nm) y atenúa la cantidad de UVB (290-320 nm) que Ilega a la superficie terrestre; en tanto que la radiación UVA (320-400 nm) y la luz visible son las más abundantes. La UVA se ha relacionado con el fotoenvejecimiento mientras que la UVB es la causante principal del eritema, la quemadura solar y los efectos carcinogénicos de las radiaciones solares..$^{1-4}$ La exposición crónica a la radiación ultravioleta causa fotoenvejecimiento, disminución de la respuesta inmunológica a patógenos ambientales e incrementa el riesgo de desarrollar neoplasias premalignas y malignas. ${ }^{1}$

\section{MEDIDAS DE PROTECCIÓN SOLAR}

Las medidas de protección solar pueden ser endógenas (constitucionales) o exógenas. La fotoprotección endógena incluye los mecanismos de protección natural contra la radiación ultravioleta (dependientes de cada individuo), en tanto que la exógena incluye las medidas físicas para evitar el daño a la exposición solar y el uso de fotoprotectores ${ }^{5}$ (Cuadro 1 ).
Marimar Saéz-de Ocariz

Ma. de la Luz Orozco-Covarrubias

Servicio de Dermatología.

Instituto Nacional de Pediatría, México.

Recibido: 4 de marzo del 2015

Aceptado: 20 de mayo del 2015

Correspondencia: Dra. Marimar Saéz-de Ocariz Servicio de Dermatología Instituto Nacional de Pediatría Insurgentes Sur 3700-C CP 04530 México, D.F.

mariadelmar71@prodigy.net.mx

Este artículo debe citarse como Saéz-de Ocariz M, Orozco-Covarrubias ML. Protección solar en el paciente pediátrico. Acta Pediatr Mex 2015;36:364-368. 
Cuadro 1. Medidas de protección de la luz solar

\begin{tabular}{|c|c|}
\hline $\begin{array}{l}\text { Fotoprotección } \\
\text { endógena }\end{array}$ & Fotoprotección exógena \\
\hline $\begin{array}{l}\text { Aumento de grosor } \\
\text { del estrato córneo } \\
\text { Reparación del ADN } \\
\text { Síntesis de moléculas } \\
\text { antioxidantes } \\
\text { Aumento en la pro- } \\
\text { ducción de mela- } \\
\text { nina }\end{array}$ & $\begin{array}{l}\text { Evitar la exposición solar, especial- } \\
\text { mente entre las } 11 \text { y las } 16 \text { horas } \\
\text { Cubrir la piel con ropas y gorros } \\
\text { adecuados } \\
\text { Usar lentes con protección solar } \\
\text { Protegerse aún en días nublados } \\
\text { Protegerse aunque la piel esté } \\
\text { bronceada } \\
\text { Uso de fotoprotectores }\end{array}$ \\
\hline
\end{tabular}

\section{Medidas físicas}

La medida más importante para prevenir los efectos de la radiación ultravioleta consiste en evitar o reducir el tiempo de exposición al sol. Los niños, por el mayor número de actividades que realizan al aire libre, reciben hasta el triple de radiación solar que los adultos. Por ello se deben evitar las actividades al aire libre entre las 11 y las 16 horas, fomentar el uso de lugares sombreados y evitar la exposición solar intencionada, incluyendo a las camas de bronceado. ${ }^{1}$

Si se va a estar expuesto al sol se debe cubrir la mayor parte posible del cuerpo con ropa. El nailon, la seda y el poliéster protegen mejor que el algodón, la viscosa, el rayón y el lino. La protección es mayor cuanto más "cerrado", más pesado y más grueso es el tejido. Deben preferirse los colores oscuros que incrementan de 3 a 5 veces el grado de protección de un tejido. ${ }^{5,6}$

Para la protección de cara y cuello se requiere el uso de gorras y sombreros, éstos proveen mayor protección cuanto mayor sea el área que cubren. Los lentes de sol protegen los ojos y las regiones perioculares y deben tener filtros solares homologados para los niños. ${ }^{1}$

\section{Protectores solares}

Los protectores solares son capaces de atenuar la radiación ultravioleta a través de dos proce- sos principales: dispersión y absorción. Estos procesos no son mutuamente excluyentes y las preparaciones comerciales pueden contener agentes que actúan a través de ambos ${ }^{1,2,4}$ (Cuadros 2 y 3 ).

Se denominan protectores solares físicos, inorgánicos o pantallas minerales aquellos que actúan como una barrera física, reflejando o dispersando la radiación ultravioleta, la luz visible y los infrarrojos. Protegen eficientemente frente a UVA y UVB; sin embargo, son cosméticamente poco aceptables. ${ }^{1,2,4}$

Cuadro 2. Ingredientes de los protectores solares

\begin{tabular}{|c|c|c|c|}
\hline \multirow[b]{2}{*}{ Tipo de compuesto } & \multicolumn{3}{|c|}{ Absorción } \\
\hline & $\begin{array}{l}\text { UVB } \\
(290- \\
320)\end{array}$ & $\begin{array}{l}\text { UVA2 } \\
(320- \\
340)\end{array}$ & $\begin{array}{l}\text { UVA1 } \\
(340- \\
400)\end{array}$ \\
\hline \multicolumn{4}{|c|}{ Químico u orgánico } \\
\hline $\begin{array}{l}\text { Derivados del ácido paraami- } \\
\text { nobenzoico } \\
\text { Ácido paraaminobenzoico } \\
\text { Padimato O }\end{array}$ & $\begin{array}{l}* * \\
* *\end{array}$ & & \\
\hline $\begin{array}{l}\text { Cinamatos } \\
\text { Octinoxato o Parsol MCX } \\
\text { Cinoxato }\end{array}$ & $\begin{array}{l}* * \\
* *\end{array}$ & & \\
\hline $\begin{array}{l}\text { Salicilatos } \\
\text { Octisalato } \\
\text { Homosalato } \\
\text { Salicilato de trotamina }\end{array}$ & $\begin{array}{l}* * \\
* * \\
* *\end{array}$ & & \\
\hline $\begin{array}{l}\text { Benzofenonas } \\
\text { Oxibenzona } \\
\text { Sulisobenzona } \\
\text { Dioxibenzona }\end{array}$ & $\begin{array}{l}* * \\
* *\end{array}$ & $\begin{array}{l}* * \\
* * \\
*\end{array}$ & * \\
\hline $\begin{array}{l}\text { Otros } \\
\text { Octocrileno } \\
\text { Ensulizol }\end{array}$ & $\begin{array}{l}* * \\
* *\end{array}$ & & \\
\hline $\begin{array}{l}\text { Avobenzona o Parsol } 1789 \\
\text { Meradimato }\end{array}$ & & $\begin{array}{l}* * \\
* *\end{array}$ & $* *$ \\
\hline Mexoryl XL & & $* *$ & $* *$ \\
\hline Tinosorb M & $* *$ & $* *$ & $* *$ \\
\hline Tinosorb S & $* *$ & $* *$ & $* *$ \\
\hline \multicolumn{4}{|c|}{ Físico o inorgánico } \\
\hline Dióxido de titanio & $* *$ & $* *$ & $* *$ \\
\hline Óxido de cinc & $* *$ & $* *$ & $* *$ \\
\hline
\end{tabular}


Cuadro 3. Algunos fotoprotectores disponibles en México

\section{Nombre}

Para niños aerosol 50+

Para niños emulsión 50+

Barra zonas sensibles 50+

Anthelios pediátrico emulsión 50+

Anthelios pediátrico aerosol $50+$

Anthelios pediátrico aerosol fácil aplicación 50+

Daylong kids loción 50+

Eclipsol baby 30

Eclipsol resistente al agua 80

Eucerin kids micropigment loción 25 (recomendado de 6 meses a 1 año)

Eucerin kids loción 50+

(mayores de 1 año)

Genovan pediátrico 40

Isdin pediatrics fotoprotector loción 50*

Isdin pediatrics fusion fluido $50+$

Isdin pediatrics aerosol transparente $50+$

Isdin pediatrics loción aerosol $50+$

Isdin pediatrics gel crema $50+$

Isdin pediatrics aerosol transparente para piel mojada 50+

Photoderm KID aerosol solar 50+

Photoderm $^{\circledast}$

Photoderm KID blue emulsión solar 50+

Prozone baby

\section{Componentes}

Avène $^{\circledast}$

Cinc y dióxido de titanio micronizados

Tinosorb M

Tinosorb S

Dióxido de titanio

Octocrileno

Anthelios ${ }^{\circledR}$

Dióxido de titanio

Mexoryl XL

Mexoryl TM

Parsol 1789

Octocrileno

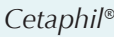

Eclipso $^{\circledast}$

Micropigmentos

Dióxido de titanio

Dióxido de titanio

Octilmetoxicinamato

Octilsalicilato

Dióxido de titanio Octilmetoxicinamato
Dióxido de titanio
Óxido de cinc
Micropigmentos
Dióxido de titanio
Tinosorb S
Octocrileno

Genovan $^{\circledR}$

Dióxido de titanio

Parsol 1789

Parsol 5000

Parsol SLX

Parsol HS

Isdin ${ }^{\circledR}$

\section{Dióxido de titanio Metoxicinamato Octocrileno}


En los protectores solares químicos u orgánicos el ingrediente activo actúa absorbiendo la radiación ultravioleta y disipando la energía como luz o calor. La mayoría absorben la radiación UVB, unos cuantos absorben la radiación entre los límites de UVA2 (320-340 nm) y sólo uno de ellos tiene una adecuada absorción en dichos límites. ${ }^{1,2,4}$

Hoy en día el factor de protección solar es la medida más utilizada para evaluar la eficacia de los protectores solares. Sin embargo, es un índice que provee información sobre UVB y no sobre UVA, por lo que se están desarrollando otras medidas de eficacia. La FDA (Food and Drug Administration) sugiere que se les denomine, de acuerdo con el grado de protección que ofrecen, factor de protección solar bajo (2-15), medio (15-30) alto (30-50) o muy alto (50+)..$^{1,2,7}$

Los protectores solares con filtros orgánicos pueden penetrar la piel en bajas cantidades (0.1 a $5 \%$ ) mientras que los que poseen filtros inorgánicos no penetran la piel, incluso con su aplicación en nanopartículas pues su penetración se limita al estrato córneo. ${ }^{8}$ Con el uso de filtros orgánicos se han descrito algunas reacciones adversas leves, fundamentalmente dermatitis por contacto o fotoalergia, principalmente con el uso de benzofenona y octilmetoxicinamato. ${ }^{9}$

La posibilidad de deficiencia de vitamina D debida al uso adecuado de protectores solares aún es controvertida, aunque la mayoría de los autores ha demostrado que el uso de protectores solares a largo plazo tiene un efecto mínimo o ninguno en las concentraciones de vitamina D o en su función. ${ }^{10-12}$ La Fundación del Cáncer de Piel (Skin Cancer Foundation) recomienda que los niños que reciben fotoprotección con regularidad complementen la dosis adecuada de vitamina D (600 UI) de fuentes alimenticias como pescado, leches fortificadas, cereales y suplementos. ${ }^{2}$
En los niños mayores de 6 meses los fotoprotectores deben tener un factor de protección mayor o igual a 25, ser de amplio espectro para protegerlos de las radiaciones UVA y UVB y ser lo más resistentes posible al agua, el sudor y el frotamiento. Hay que aplicarlos de forma generosa y uniforme en todas las áreas expuestas (no protegidas por la ropa) todos los días del año, 15 minutos antes de la exposición al sol y volverlos a aplicar cada 2 a 4 horas, en particular después de nadar, frotarse con una toalla o sudar excesivamente. ${ }^{1,2}$

En los menores de 6 meses de edad no se recomienda la aplicación de fotoprotectores para evitar posibles problemas de toxicidad. Este grupo de edad tampoco debe exponerse directamente al sol sin ropa protectora, pues tiene menor capacidad de producción de melanina y de sudoración. También se recomienda limitar las exposiciones solares en niños menores de 3 años de edad. ${ }^{1,2}$

\section{Aspectos educativos}

Las medidas de fotoprotección se aconsejan en todas las edades, pero deben ser más intensas en la población infantil y juvenil dado que $80 \%$ de nuestra exposición al sol tiene lugar antes de los 18 años, ${ }^{13}$ por lo que el uso adecuado de protectores solares durante la infancia y la adolescencia puede reducir la incidencia de cáncer de piel no melanoma en cerca de $80 \%{ }^{14}$

La mayoría de las campañas de protección a los rayos solares van dirigidas a los niños porque se ha demostrado que las prácticas que evitan o minimizan la exposición deben comenzar lo más temprano posible para hacerlas un hábito, pues los comportamientos que se adquieren de forma temprana tienden a perdurar durante la vida con mayor intensidad. ${ }^{15,16}$ El grupo de los adolescentes es particularmente importante porque los estudios confirman que, mientras 
los conocimientos sobre fotoprotección van aumentando con la edad, los comportamientos se van deteriorando y señalan la necesidad de cambiar las actitudes hacia la exposición solar y el bronceado. ${ }^{15,16}$

Para lograr una disminución en la incidencia de cáncer de piel es necesario promover la fotoprotección entre los padres (y sobre todo entre los niños) mediante campañas educativas que incrementen el conocimiento de las medidas de fotoprotección y que favorezcan cambios en nuestro comportamiento respecto de la exposición al sol. En esta labor educativa los pediatras y los dermatólogos tienen un papel fundamental.

\section{REFERENCIAS}

1. Valdivieso-Ramos M, Herranz JM. Actualización en fotoprotección infantil. An Pediatr (Barc) 2010;72(4):282.e1-282. e9

2. Quatrano NA, Dinulos JG. Current principles of sunscreen use in children. Curr Opin Pediat 2013;25(1):122-9.

3. Wilson BD, Moon S, Armstrong F. Comprehensive review of ultraviolet radiation and the current status on sunscreens. J Clin Aesthet Dermatol 2012;5(9):18-23.

4. González S, Fernandez-Lorente M, Gilaberte-Calzada $Y$. The latest on skin photoprotection. Clin Dermatol 2008;26(6):614-26.

5. Gilaberte Y, Coscojuela C, Saenz de Santamaria MC, González S. Fotoprotección. Actas Dermosifiliogr 2003;94(5):27193.
6. Hoffman K, Laperre J, Avermaete A, Altmeyer P, Gambichler T. Defined UV protection by apparel textiles. Arch Dermatol 2001;137(8):1089-94.

7. Koshy JC, Sharabi SE, Jerkins D, Cox J, Cronin SP, Hollier LH Jr. Sunscreens: evolving aspects of sun protection. J Pediatr Health Care 2010;24(5):343-6.

8. Sadrieh N, Wokovich AM, Gopee NV, Zheng J, Haines D, Parmiter $\mathrm{D}$, et. al. Lack of significant dermal penetration of titanium dioxide from sunscreen formulations containing nano- and submicron-size Ti02 particles. Toxicol Sci 2010;115(1):156-66.

9. Haylett AK, Chiang YZ, Nie Z, Ling TC, Rhodes LE. Sunscreen photopatch testing: a series of 157 children. Br J Dermatol 2014;171(2):370-5.

10. Norval M, Wulf HC. Does chronic sunscreen use reduce vitamin $\mathrm{D}$ production to insufficient levels? $\mathrm{Br} J$ Dermatol 2009;161(4):732-6.

11. Linos E, Keiser E, Kanzier M, Sainani KL, Lee W, Vittinghoff E. Sun protective behaviors and vitamin D levels in the US population: NHANES 2003-2006. Cancer Causes Control 2012;23(1):133-40.

12. Kannan S, Lim HW. Photoprotection and vitamin D: a review. Photodermatol Photoimmunol Photomed 2014;30(23):137-45

13. Pustisek N, Sikanic-Dugic N, Hirsl-Hecej V. Acute skin sun damage in children and its consequences in adults. Coll Antropol 2010;34(Suppl 2):233-7.

14. Heckman CJ, Coups EJ. Correlates of sunscreen use among high school students: a cross-sectional survey. BMC Public Health 2011;11:679-87.

15. Dusza SW, Halpern AC, Satagopan JM, Oliveria SA, Weinstock MA, Scope $A$, et. al. Prospective study of sunburn and sun behavior patterns during adolescence. Pediatrics 2012;129(2):309-17.

16. Lin JS, Eder M, Weinmann S. Behavioral counselling to prevent skin cancer: a systematic review for the U.S. Preventive Services Task Force. Ann Intern Med 2011;154(3):190-201. 